\title{
Ability of Lung Specialist to Predict a Diagnosis of Lung Cancer during the First Visit to a Fast Track Clinic
}

\section{K.A. Gaber ${ }^{1 *}$, S.E. DeBarr ${ }^{2}$, S. Stevens ${ }^{3}$, A. Elkkari ${ }^{4}$, H Bakere $^{5}$ and N.J. Withers ${ }^{6}$}

${ }^{1}$ Department of Respiratory Medicine, BMC, Benghazi University, Libya

${ }^{2}$ Department of Medicine, Southmead Hospital, Bristol, UK

${ }^{3}$ Department of Respiratory Medicine, Royal Devon and Exeter Hospital, Exeter, UK

${ }^{4}$ Heart and Lung Unit, Torbay Hospital, Torquay, UK

${ }^{5}$ Department of Respiratory Medicine, Truro Hospital, Truro, UK

${ }^{6}$ Department of Respiratory Medicine, Royal Devon and Exeter Hospital, Exeter, UK

\section{Introduction}

Lung cancer remains a major medical problem. It is the commonest cause of cancer death in males and the second most common in females with an annual death rate of approximately 29,000 in England and Wales [1]. There has been no improvement in the 5-year survival rate of lung cancer for the last 30 years [1]. In April 2000, as part of the Cancer Services Standards, the Department of Health issued guidelines for patients with suspected lung cancer to be seen by a lung cancer specialist within 2 weeks of GP referral [2]. We wanted to assess how accurate specialist hospital doctors are at predicting lung cancer during the initial consultation with the patient.

\section{Method}

We prospectively studied all patients referred to the lung cancer fast track clinic, under the 2-week waiting time recommendation, in the period from April to August 2004 in 3 District General Hospitals in the South West of England. Following the initial consultation in outpatients, the assessing doctors were asked to complete an A4 sized proforma, in tick-box format. The following information was collected: (1) grade of assessing doctor (2) presence or absence of the following symptoms: cough for more than 3 weeks, increased shortness of breath, chest pain, weight loss, hoarse voice and haemoptysis (subdivided into single, recurrent episodes or flecks) (3) history of smoking or asbestos exposure and (4) the presence or absence of clubbing or Horner's syndrome. The doctors were then asked to indicate whether they thought the diagnosis of lung cancer was "likely" or "unlikely", based solely upon their initial assessment, and before further investigations, such as CT scan or bronchoscopy were performed. In addition the assessing lung specialist was asked to interpret an up-to-date chest radiograph as (a) suggestive of cancer; (b) cancer needs to be excluded or (c) normal. All patients were followed up until a diagnosis of lung cancer was confirmed or for a period of 18 months if cancer was not confirmed. A diagnosis of lung cancer was deemed positive if a) a positive histology result was obtained or b) a clinical diagnosis supported by discussion at a MultiDisciplinary Team Meeting (MDT) was reached. All data collected was blinded before analysis.

\section{Results}

168 proformas were received from the 3 hospitals. 14 were excluded due to incomplete data. 75 patients were predicted by the assessing doctors as "likely" to have lung cancer. The final diagnosis was documented in 70 of these patients ( 44 seen by consultants, 18 by

\begin{tabular}{|l|c|c|c|}
\hline & All Group $(\mathrm{n}=70)$ & $\begin{array}{c}\text { Seen by Consultant } \\
(\mathrm{n}=44)\end{array}$ & $\begin{array}{c}\text { Seen by SpR } \\
(\mathrm{n}=18)\end{array}$ \\
\hline $\begin{array}{l}\text { "Likely" lung cancer } \\
\text { and correct diagnosis }\end{array}$ & $53(76 \%)$ & $33(75 \%)$ & $13(72 \%)$ \\
\hline $\begin{array}{l}\text { "Likely" lung cancer } \\
\text { and incorrect diagnosis }\end{array}$ & $17(24 \%)$ & $11(25 \%)$ & $5(28 \%)$ \\
\hline
\end{tabular}

Tables 1a: Accuracy rate for predicting the likelihood of lung cancer.

\begin{tabular}{|l|c|c|c|}
\hline $\begin{array}{l}\text { "Likely" lung cancer and "sugges- } \\
\text { tive" chest radiograph }\end{array}$ & $\begin{array}{c}\text { All Group } \\
(n=53)\end{array}$ & $\begin{array}{c}\text { Seen by Consul- } \\
\text { tant }(n=32)\end{array}$ & $\begin{array}{c}\text { Seen by SpR } \\
(n=14)\end{array}$ \\
\hline $\begin{array}{l}\text { "Likely" lung cancer and "sugges- } \\
\text { tive" chest radiograph and correct } \\
\text { diagnosis }\end{array}$ & $43(81 \%)$ & $25(78 \%)$ & $11(79 \%)$ \\
\hline
\end{tabular}

Tables 1b: Accuracy rate for predicting the likelihood of lung cancer.

specialist registrars and 8 by others). 18 had squamous cell carcinoma, 10 adenocarcinoma, 2 small cell carcinoma, 1 mesothelioma, 14 other tumours and 8 clinical diagnoses supported by MDT decisions. In 53 (76\%) the doctor's prediction was correct (Table 1a).

The chest radiographs were interpreted as "suggestive of cancer" in 61 cases, of which, 53 (87\%) were also thought to have "likely" lung cancer. 43 (81\%) had a correct final diagnosis.

44 out of 104 patients seen by the consultants were deemed as "likely" lung cancer. 33 (75\%) had lung cancer. 32 patients seen by the consultant were thought to have both "likely" lung cancer and a chest radiograph "suggestive of cancer". 25 (78\%) had lung cancer (Table 1b). 18 out of 45 patients seen by the specialist registrars were deemed as "likely" lung cancer. 13 (72\%) had lung cancer. 14 patients seen by the specialist registrars were thought to have both "likely" lung cancer and a chest radiograph "suggestive of cancer". 11 (79\%) had lung cancer (Table 1b).

Smoking history was documented in 62 out of the 70 cases thought to be "likely" lung cancer. 37 were ex-smokers, 18 current smokers and 7 never smoked. The associated presenting symptoms and signs of the patients who were thought "likely" to have lung cancer and had a correct diagnosis of lung cancer, were compared with those who were thought to be "likely" to have lung cancer and had an incorrect diagnosis. Hoarse voice and clubbing were only seen in patients who were deemed "likely" to have lung cancer and had a correct diagnosis. Weight loss was reported in $40 \%$ of patients who were deemed "likely" to have lung cancer and had a correct diagnosis, compared with $12 \%$ in patients who were deemed "likely" to have lung cancer but did not. For other associated symptoms see Table 2.

79 patients were deemed "unlikely" to have lung cancer by the assessing doctors. Follow up results at 18 months were available for 69

*Corresponding author: K.A. Gaber, Department of Respiratory Medicine, BMC Benghazi University, Libya, UK, E-mail: khalidgbr@doctors.net.uk

Received November 10, 2011; Accepted April 21, 2012; Published April 23, 2012

Citation: Gaber KA, DeBarr SE, Stevens S, Elkkari A, Bakere H, et al. (2012) Ability of Lung Specialist to Predict a Diagnosis of Lung Cancer during the First Visit to a Fast Track Clinic. J Pulmon Resp Med S5:004. doi:10.4172/2161-105X. S5-004

Copyright: ( 2012 Gaber KA, et al. This is an open-access article distributed under the terms of the Creative Commons Attribution License, which permits unrestricted use, distribution, and reproduction in any medium, provided the original author and source are credited. 


\begin{tabular}{|l|c|c|}
\hline $\begin{array}{l}\text { Symptoms and } \\
\text { Signs }\end{array}$ & $\begin{array}{c}\text { Percentage of patients } \\
\text { thought "likely lung cancer", } \\
\text { with positive diagnosis }\end{array}$ & $\begin{array}{c}\text { Percentage of patients } \\
\text { thought "likely lung cancer" } \\
\text { with negative diagnosis }\end{array}$ \\
\hline Haemoptysis & $36 \%$ & $24 \%$ \\
\hline - Whole & $9 \%$ & $6 \%$ \\
\hline - Single & $17 \%$ & $18 \%$ \\
\hline - Recurrent & $4 \%$ & $0 \%$ \\
\hline - Flecks & $6 \%$ & $0 \%$ \\
\hline - Unspecified & $62 \%$ & $71 \%$ \\
\hline Cough & $53 \%$ & $71 \%$ \\
\hline Increasing SOB & $9 \%$ & $29 \%$ \\
\hline Asbestos exposure & $40 \%$ & $12 \%$ \\
\hline Weight Loss & $36 \%$ & $24 \%$ \\
\hline Chest Pain & $9 \%$ & $0 \%$ \\
\hline Hoarse Voice & $17 \%$ & $0 \%$ \\
\hline Clubbing & & \\
\hline
\end{tabular}

Table 2: Symptoms \& signs and the percentage of patients with positive \& negative diagnosis>

(87\%) of these. 5 out of $69(7 \%)$ were found to have lung cancer (2 adenocarcinoma, 1 large cell carcinoma, 1 non small cell carcinoma, 1 MDT decision).

\section{Discussion}

It is human nature to try to predict the outcome of a given problem and this is subject to human error. The individual's judgement is usually affected by variable factors such as available information and personal experience. Diagnosis of lung cancer continues to be a challenging process, which requires clinical assessment in addition to invasive and non-invasive investigations. There have been studies, which have looked at the prediction accuracy of certain aspects in the management of lung cancer. In an attempt to predict the prognosis of terminally ill patients, doctors were found to be inaccurate in most cases [3]. In a prospective cohort study involving 343 doctors providing an estimate for 468 terminally ill patients, at the time of hospice referral, only 20 $\%$ of predictions were accurate and $63 \%$ were over optimistic [3]. In a recent systematic review of physicians' efforts to predict terminally ill cancer patients' outcome, survival was consistently overestimated in 7 out of 8 studies [4]. Doctors also tried to predict patients' satisfaction of medical care and were correct in only $20 \%$ of cases [5].

Given the same circumstances, significant differences of opinion have been noted between different members of the medical team. Predicting the futility of medical interventions, nurses were found to be more pessimistic in general, but were more often correct than doctors in the judgements of dying patients [6].

Not all predictions have been disappointing. In assessing elderly patients' views on cardiopulmonary resuscitation, doctors were inaccurate in only $24 \%$ of cases [7] and they were found to be correct in 94.5\% of cases when predicting intensive care unit treatment outcomes [8]. Doctors were also good in predicting the duration of certified sickness absence (predicting return to work), being correct in $84 \%$ of short-standing episodes [9].

The question we tried to answer was how good respiratory doctors are at predicting lung cancer after taking history, examining the patient and interpreting the chest radiograph. The overall diagnostic accuracy of respiratory doctors was $76 \%$ when lung cancer was deemed "likely". This improved to $81 \%$ when the assessing doctor also interpreted the chest radiograph as "suggestive of cancer". There was no significant difference in the overall accuracy rate between consultants and specialist registrars $(75 \%$ vs. $72 \%$, respectively). In those patients deemed "unlikely" to have lung cancer, doctors were correct in $93 \%$ of cases.

When the assessing doctor thought the diagnosis of lung cancer was "likely", the presence of clubbing or hoarse voice was associated with $100 \%$ prediction accuracy. The occurrence of weight loss made the correct prediction of lung cancer 3 times more likely, while cough, increased shortness of breath and haemoptysis had no significant influence.

Although it is generally perceived by patients and their General Practitioner that haemoptysis has an important association with the diagnosis of lung cancer, previous work showed that this is not the case [10]. Since the commonest cause of haemoptysis is idiopathic [11], and due to the low threshold of referring high-risk patients with this symptom to the Fast Track Clinic, it is not surprising that haemoptysis is not a good predictor of diagnosis of lung cancer.

It is interesting to observe that doctors are more accurate in negatively predicting lung cancer than positively predicting it. This might reflect doctors' caution not to miss a possible diagnosis of lung cancer. It is a happier outcome for doctors and patients to have an incorrect positive prediction of lung cancer than an incorrect negative one.

\section{Conclusion}

"Doctor, have I got cancer?" In the current era of internet culture, this question and many more are being asked with an increasing frequency by patients during their first consultation at the Fast Track Clinic. Many doctors are reluctant to predict an answer to those questions, [12] partially due to lack of supportive data. This study would help the assessing doctors in the Fast Track Clinic to provide an answer that is likely to be correct in $76 \%$ of cases if the doctor thought lung cancer was the likely diagnosis, and $93 \%$ when the diagnosis of lung cancer was considered "unlikely". It is important to recognise that in a quarter of cases in the "likely" lung cancer group, the doctor's prediction was incorrect. To our knowledge this study has not been performed before. It would also be interesting to compare the prediction accuracy of lung specialists with that of the referring general practitioners.

Although it is our duty not to withhold information from patients this should be given in an optimistic way [12]. Balancing the information given to patients with suspected lung cancer is an important skill doctors should exercise during the first clinic consultation.

\section{References}

1. No Author Listed (2005) The Diagnosis and Treatment of lung cancer. NICE Guidelines.

2. No Author Listed (2000) Cancer referral guidelines. Referral Guidelines for Suspected Cancer. Department of Health.

3. Christakis NA, Lamont EB (2000) Extent and determinants of error in doctors prognoses in terminally ill patients: prospective cohort study. BMJ 320: 469-472.

4. Glare P, Virik K, Jones M, Hudson M, Eychmuller S, et al. (2003) A systematic review of physician's survival predictions in terminally ill cancer patients. BMJ 327 195-198.

5. Merkel WT (1984) Physician prediction of patient satisfaction. Do doctors know which patients are satisfied? Med Care 22: 453-459.

6. Frick S, Uehlinger DE, Zuercher Zenklusen RM (2003) Medical futility: predicting outcome of intensive care patients by nurses and doctors-a prospective comparative study. Cit Care Med 31: 456-461.

7. Krumholz HM, Phillips RS, Hamel MB, Teno JM, Bellamy P, et al. (1998) Resuscitation preference among patients with severe congestive heart failure. Results from the SUPPORT project. Study to understand prognosis and preference for outcome and risks of treatments. Circulation 98: 648-655. 
Citation: Gaber KA, DeBarr SE, Stevens S, Elkkari A, Bakere H, et al. (2012) Ability of Lung Specialist to Predict a Diagnosis of Lung Cancer during the First Visit to a Fast Track Clinic. J Pulmon Resp Med S5:004. doi:10.4172/2161-105X.S5-004

8. Hahnel J, Friesdorf W, Schwilk B, Marx T, Blessing S (1992) Can a clinician predict the clinical equipment a patient will need during an intensive care unit treatment? An approach to standardise and redesign the intensive care unit workstation. $J$ Clin Monit 8: 1-6.

9. Reiso H, Gulbrandsen P, Brage S (2004) Doctors' prediction of certified sickness absence. Fam Pract 21: 192-198.
10. Le June LR, Lewis NR, Baldwin DR (2002) Presenting symptoms and chest radiographic features in urgent referrals for suspected lung cancer: how well do they discriminate? Thorax (s).

11. Hirshberg B, Biran I, Glazer M, Kramer MR (1997) Hemoptysis: etiology, evaluation and outcome in a tertiary referral hospital. Chest 112: 440-444.

12. Ubel PA (2001) Truth in the Most Optimistic Way. Ann Intern Med 134: 1142-1143.

This article was originally published in a special issue, Lung Cance Challenges to the future handled by Editor(s). Dr. Ramon Andrade de Mello, University of Porto, Portugal 